\title{
The Progression of Life: Before, Now, and In the Future; Here and Elsewhere
}

\author{
Daniel Goldman \\ contact@danielgoldman.us \\ 0000-0003-2835-3521
}

June 2020

\section{Abstract}

Evolution and abiogenesis are usually considered two different topics in biology. However, recent work on the relationship between thermodynamics and life, as well as complexity and evolution, suggest that these topics may all be intimately related. Leveraging novel research on thermodynamics and complexity theory, I attempt to show that thermodynamics seems to exert a universal selective pressure (entropic pressure). This pressure seems to exist on the atomic and molecular level, biological level, and even societal level, and results in a number of interesting consequences. There is still much work to do in order to increase our understanding of the relationship between thermodynamics, complexity theory, abiogenesis, and evolution. Understanding entropic pressure may allow for robust predictions about life in the past and the potential nature of life in the future, and help us better predict the nature and scarcity of life beyond this world. 


\section{Introduction}

There is a lot to be said about life and its evolution. Life is notoriously difficult to define and numerous attempted definitions exist. Steven A. Benner summarizes much of the question about the definition of life in a 2010 article on the topic. Some scholars such as Daniel Koshland, simply gave a list of properties that life seems to have. On the other hand, Carl Sagan related life to self-sustaining chemical systems and Darwinian evolution. One inherent problem in defining life is that we have only the examples found on this planet. Benner admitted that while the "NASA definition" appears fine so far, if we met a truly alien entity, such as one made of pure energy, we would need to rethink the definition. [3] Even still, we can learn more about the nature of life by learning about the boundaries between non-life and life, as we know it.

As Carl Sagan defined life in terms of Darwinian evolution, it appears that we may need to understand evolution in order to understand life. At its most fundamentalism level, evolution is simply any change in a system over time. However, evolution usually refers to biological processes. The body of theory surrounding biological evolution is one of the most robust bodies of theory that exists. And yet it is still lacking in certain ways. Definitions of biological evolution are often vague and problematic. The common reliance on the concept of speciation makes definitions of evolution weaker. Meanwhile, a failure to find a unifying theory between evolution and abiogenesis is troubling. In some ways, the separation between theories on the origin of life and the theories on the evolution of life are as concerning as the separation between quantum mechanics and general relativity. This paper considers novel theories in statistical physics related to self-replication, as well as theories in complexity theory related to self organization, and addresses how they may be useful in the process of integrating theories of evolution and abiogenesis, and in the study of exobiology and synthetic life. This paper is a heavy revision of an earlier paper on this topic 7 .

\section{Some Issues with Defining Evolution}

Because tackling the concept of life appears to require dealing with evolution, I will start the discussion here. This section of the paper is not an attempt to define evolution, but rather instead constitutes a critique of some existing definitions of evolution and issues with perception on how evolution works. "Evolution is a change in allele frequency over time 8;" this definition seems nice enough, but there are a few issues with it. First, while it is not likely to occur, allele frequencies could flip back and forth during each generation. While this situation is indeed change in allele frequency over time, it may not be what people generally consider to be evolution.

However, there is a more clear cut example that can be conceived of in a thought experiment. It is not uncommon for plants to have offspring with double the chromosome count from the original. This process is known as polyploidy. In polyploidy, the entire genome is duplicated[4]. However, the genome is far 
from identical to the parent genome. It has twice as many chromosomes: it is twice as large. It is hard to argue that this condition is not an example of evolution, especially since polyploid offspring can be completely fertile, but not capable of reproducing with members of the parent species. It is incredibly rapid evolution. And yet, depending on the reason for the genome duplication, it is possible to have offspring that have the exact same allele frequency as the parent.

As an extreme example, a plant could exist entirely as a clonal population, where each individual plant is a genetic clone. If polyploidy were to occur in such a population, it could result in a new lineage, with the exact same allele frequency, but twice the genome size. Therefore even though there is evolution, the allele frequency of the population has not changed in this thought experiment. So change in allele frequency over time does not quite suffice, but there is another major concern with the way evolution is often defined. Specifically, relying on the concept of a species in a definition of evolution. While people will continue to abuse scientific concepts to argue their positions, science communication should be as clear as possible, from the beginning. Speciation, microevolution, and macroevolution are terms that are often used in creationist debate, and unfortunately they stem from a misunderstanding. There is no such thing as microevolution and macroevolution. One of the reasons for the misconception is in our desire to label and group. This desire has resulted in a flawed understanding of species and speciation. It gives the impression that evolution occurs when one species becomes another, but there is no clear delineation for species, and evolution is a constant process that occurs along a population's lineage.

However, if the concept of a species were valid, then species inclusion should be transitive. That is to say that if you have three populations, A, B, and C, then if $\mathrm{A}$ is the same species as B, and B is the same species as C, then A should be the same species as $\mathrm{C}$. So consider one of the major concept of a species: two organisms belong to the same species if they can reproduce and create viable offspring. But the concept of a ring species makes this criteion problematic. In a ring species, there can be three or more populations, in which some of the populations can interbreed and produce viable offspring, but populations which

are genetically or physically further away from one another produce offspring that are not viable or which fail to produce offspring at all.

\section{Linking Evolution and Abiogenesis Using Ther- modynamics and Complexity}

The attempt to unify theories of evolution and abiogenesis is not new. Nor is the attempt to help explain biological systems using thermodynamic properties. But many have rejected a connection between theories on non-equilibrium thermodynamics and life 10. However, research conducted by Jeremy England suggests that matter, or really any collection of units that can be thermodynam- 
ically driven, will tend towards a form of self replicating order, when exposed to a heat bath 5 . Bar-Yam et al. take a different approach to understanding organization of unorganized systems, and come to a similar conclusion. They refer to the tendency of unordered systems to be unstable with respect to order, within an open system, as an "inverse second law of thermodynamics." [2] And so when taken together, an interesting result is that entropy, in an open system, seems to act as the inverse of its own dynamic, thus driving systems to become ordered, and more importantly for the discussion of abiogenesis, self replicating systems.

England's work suggests that "the likely evolutionary outcomes are going to be the ones that absorbed and dissipated more energy from the environment's external drives on the way to getting there 5 ." However, England's work focuses on the molecular level. He does not attempt to argue that entropy in an open system actually results in abiogenesis. But what makes me hopeful of a grand unification theory of evolution and abiogenesis, and that thermodynamics will be this source of this grand unification, is that while England's work focuses on basic matter, Bar-Yam et al. focused on social organization. And while the approaches were entirely different, the conclusion was essentially the same.

Additionally, in order to more easily discuss this concept in the future, I would like to suggest the use of a new term, entropic pressure, which seeks to capture the essence of the dynamics described in this research. It is entropic pressure that I hope will eventually act as a form of fundamental law of evolution and life. However, to define a law, a few criterion should be met. The law must be concise, it must act as a foundation for a large body of theory, and it must be well tested. That thermodynamics is closely related to biological law is again not a new concept. Trevors and Saier suggest that the first law of biology should be that "all living organisms obey the laws of thermodynamics 15]." But all of the matter and energy in the universe obeys thermodynamics, and so if the laws of thermodynamics are valid, then it is an obvious result that life must obey thermodynamics. Discussing a relationship between thermodynamics and life only makes sense if a direct and insightful link between the two can be elucidated. Until recently, exactly how life is influenced by thermodynamics has not been clear. But entropic pressure gives us a connection.

\subsection{Mathematical Integration}

Bar-Yam et al. and England look at two seemingly different concepts, the former looking large scale organization and complexity, and England looking at the smallest scale of atomic and molecular actions, driven by entropy. And yet complexity theory and thermodynamics both seem to yield similar results. It may be that the "inverse second law" that Bar-Yam et al. considered is not an alternative law at all, but rather a different perspective of the same fundamental principle. It might therefore be useful to see if a more concise mathematical representation of this inversion of the second law can be developed, integrating both complexity theory and thermodynamics.

But how can these two seemingly disconnected areas of study be integrated 
and what can we learn from them? In Introduction to Cybernetics, W. Ross Ashby develops a concept of requisite variety, where variety is the number of possible states in the system[1]. An important feature of his work is that "a set's variety is not an intrinsic property of the set: the observer and his powers of discrimination 1. " Bar-Yam et al. (2018) relies on a multiscale variant[2]. Is there an analog to the observer discrimination factor within England's work? It seems so. The property of self-replication is observer driven: it requires developing a scheme to quantify how many copies of a potential replicator exist [5].

\section{Direction of Evolution}

The idea of entropic pressure leads to an interesting result: evolution has a direction. And yet, it is often said that evolution has no direction. Much of this section is philosophical in nature. However, as this paper delves into questions of the very nature of life, there is really no benefit in trying to avoid these issues.

\subsection{Jaded by Creationism}

In many cases, this comment is a response to creationism and intelligent design. However there are epistemological issues with making such claims. As such, this section of the paper is as much about the philosophy of science and science communication as it is about the science of evolution. It is difficult to comment on what is and is not appropriate discussion in science, without tackling the nature of science. There are a few different views of science, but there is one common element that all forms of science have: science is involved in that which we can investigate empirically. Religion is not. And one could argue that religion is specifically involved in that which lacks a known method of empirical investigation. Stephen Jay Gould called the separation of science and religion non-overlapping magisteria, or NOMA 14 . Though, even with this concept, one can influence the other. As our understanding of the world, through science expands, it is not uncommon to see religions take these new ideas into account. Furthermore, scientists may be influenced to an extent by their own religiosity. As long as the fundamental tenets of science are maintained, such as letting evidence lead and refusing to make statements of truth when there is no empirical evidence, we can maintain the validity of scientific investigation, even under this condition. Unfortunately when it comes to evolution, these tenets seem to be violated repeatedly.

With that brief discussion of the philosophical boundaries of science out of the way, I will begin my argument in earnest. As I already mentioned, science is about making claims based on evidence. If there is no evidence, we should not be making claims. We may utilize assumptions in our theories, but an assumption is not a claim. Yet when it comes to claims about the direction of evolution, there is a propensity to ignore the limits of science, and simply claim that these statements are true. There may be a variety of reasons why this is the case. One reason is because there tends to be an outright rejection of 
manipulation of evolutionary dynamics by deities or similar agents. However, the existence of such entities are beyond the scope of scientific investigation. Furthermore, parsimony and other roles for theory selection suggest that if there is no evidence suggesting the need for an added layer, or if that added layer is unfalsifiable, the layer should be excluded. Therefore current evolutionary theory does not include a concept of direction. But that is very different from including a layer suggesting that there is no direction. That addition too violates scientific methodology.

There are a number of reasons why the tendency to state that evolution is directionless exists. In the past, the concepts of more evolved and less evolved were used to treat large groups of people in very inhumane ways. Additionally, directed evolution often refers to evolution that is directed by some intelligent designer, such as a god. But these issues do not excuse biologists and evolutionary scientists for the certainty with which they discuss these topics.

\subsection{Directors and Directorless Direction}

Now, it is really enough to point out that we have not falsified the existence of a divine intervener or a mundane process by which evolution is driven in an overall direction, and as an atheist and a scientist, I cannot really address the existence of a divine mover, but I will provide two potential forces in evolution, one which involves agency, and one which does not, but could lead to the concept of "direction" in evolution. The first is sexual selection, and the second is based on consequences of the second law of thermodynamics.

Without any limit to what could evolve, and without anything driving evolution in a certain direction, evolution would be nothing more than a complicated form of a random walk. But there are a number of recognized driving forces that direct evolution and some even have a degree of agency. One driving force of evolution, which has at least some level of agency is sexual selection. This form of selection is not a result of an external factor, but factors internal to the decision making process of the animals that are selecting mates. There are conscious decisions made in selecting preferential mates all the time. Sexual selection is not purely random. Humans may do this to the greatest degree, but many other species pick and choose which mate they want. Of course, this process is guided by pre-wired cognitive processes which have evolved over time, and so agency driving evolution itself is also in part driven by past evolutionary results. But this does not change that organisms actively drive evolution in one direction or another. Such a process is not blind.

But if entropic pressure is a valid law, then there is yet another director, and it is universal. While there are still many different branches evolution could take, entropic pressure drives evolution in a fairly predictable fashion. One benefit of this law is that evolutionary theory can move into the realm of prospective analysis, rather than simply retrospective questioning. Much of evolutionary theory is focused on what has happened in the past. It is primarily the study of how organisms alive today came to be. There is little in evolutionary theory that allows us to predict what will come next, or even what would evolve elsewhere 
in the universe.

One might ask, "why should complex multi-cellular organisms, let alone whole societies evolve on other worlds?" Entropic pressure suggests that this result is almost a foregone conclusion. England's research does not specify the level on which entropy is acting. It suggests that any collection of "units" which can self arrange, will tend to do so. Thus matter will tend to self arrange into self replicating life forms. These self replicating life forms themselves, being units, will tend to form into self replicating collections. Thus the evolution from unicellularity to multicellularity is not something which should be unique or rare.

\section{Life Beyond Our Home}

It is difficult to study life beyond our world, in part because we have no ability to reach other planets. All research, as of now, needs to be indirect. However, if the idea of entropic pressure is correct, there are some rather significant implications for exobiology. Among other benefits, it offers insight into estimating parameters of the Drake Equation. There are a number of parameters that are used within the Drake Equation, including $f_{l}, f_{i}$, and $f_{c}$, which are the fraction of habitable planets that develop life at some point, the fraction of which become intelligent, and the fraction of those which develop the capacity to release signals that can be detected 16 .

In "Dissolving the Fermi Paradox," authors Sandberg et al. suggest that uncertainties in parameters of the Drake Equation can drastically increase the probability of there being no other intelligent life in the observable universe. The results of the paper rely on high levels of uncertainty, starting with the uncertainty of abiogenesis. 11. However, England et al. have produced results that seem to suggest that processes leading to abiogenesis are indeed likely to occur. It may be possible to take their work and use it more directly in calculating probabilities, thus reducing uncertainty considerably.

Moroever, as already mentioned, whether looking at the problem from a perspective of thermodynamics or from the perspective complexity theory, the general behavior appears to be the same regardless of the level at which the analysis is being conducted. While the specific physics involved may vary, one could say that the transitions from molecular to unicellular, from unicellular to multicellular, and from multicellular to societal organism, are topologically identical. Therefore, $f_{l}, f_{i}$, and $f_{c}$ are dependent parameters and are dependent on a more fundamental mechanism.

\subsection{Using What We Have}

Since this article includes a discussion of the here and now, it might be useful to bring into consideration the following question: how many data points do we have? For instance, Howard A. Smith suggests that it is important to consider that we may be alone in the universe, or at least effectively alone 13. He defines 
intelligent life as "life able to communicate between stars 13." It makes sense that if we're talking about life on other worlds, we are effectively alone if there is no other example of such life. But are we even alone on Earth? Recent analysis has suggested that human and cetacean brain evolution appear to follow similar paths and that this evolutionary trajectory is related to pressures that arise from social cooperation 6 .

We can also generate more data points by creating life ourselves. Synthetic biology can be broken down into three types of engineering practices: those involving the manipulation and creation of genetic circuits, those involving the manipulation and creation of entire genomes, and those involving the manipulation and creation of living organisms themselves 9 . And so we do not necessarily have to wait until we find examples of life that do not fit our current theories of its nature. And by developing such examples of artificial life, we may very well be able to better understand the boundary between non-life and life 9 .

However, without some understanding already, we are in many ways poking around in the dark. This issue is again where thermodynamic theories on life can aid the development of synthetic life, so that it can in turn aid in furthering our understanding of life itself. For instance, using chemical toy chemical models, it appears that self-replication is dominated by cooperation of interconnected systems, and so creating an environment that favors these systems over isolated autocatalytic cycles is preferable, even though doing so is an admittedly difficult $\operatorname{task} 12$.

\section{Conclusion}

Evolution is a complex topic, and one normally separated from abiogenesis. However, entropic pressure suggests that that the two concepts are fundamentally related. Entropic pressure suggests that, contrary to common assertions, evolution does have a direction. Thus, exploring how entropy functions in open systems may allow us to create a fully unified theory on life. Moreover, thermodynamics and complexity theory appear more closely related than some might consider them to be. Both bodies of theory seem to suggest that the universe favors systems of increasing order and complexity, even as the universe as a whole moves towards greater disorder. This theory may help us predict, in general terms, how life would evolve on other worlds, and how likely it is that life has evolved on those worlds. Finally, this theory, along with experimentation on synthetic biology, may yield benefit for each other. 


\section{References}

[1] W. Ross Ashby. An introduction to cybernetics. English. OCLC: 960267567. 2015. ISBN: 9781614277651.

[2] Taeer Bar-Yam, Owen Lynch, and Yaneer Bar-Yam. "The Inherent Instability of Disordered Systems". In: (2018). eprint: arXiv:1812.00450.

[3] Steven A. Benner. "Defining Life". In: Astrobiology 10.10 (Dec. 2010), pp. 1021-1030. DOI: 10.1089/ast.2010.0524. URL: https://doi.org/ 10.1089/ast.2010.0524.

[4] Richard C. Cronn, Randall L. Small, and Jonathan F. Wendel. "Duplicated genes evolve independently after polyploid formation in cotton". In: Proceedings of the National Academy of Sciences 96.25 (1999), pp. 1440614411. ISSN: 0027-8424. DOI: 10.1073/pnas.96.25.14406 eprint: https: //www . pnas . org / content/96/25/14406 . full ·pdf. URL: https : //www.pnas.org/content/96/25/14406

[5] Jeremy L. England. "Statistical physics of self-replication". In: The Journal of Chemical Physics 139.12 (2013), p. 121923. DOI: 10.1063/1. 4818538, eprint: https ://doi.org/10.1063/1.4818538, URL: https : //doi.org/10.1063/1.4818538.

[6] Kieran C. R. Fox, Michael Muthukrishna, and Susanne Shultz. "The social and cultural roots of whale and dolphin brains". In: Nature Ecology 83 Evolution 1.11 (Oct. 2017), pp. 1699-1705. DOI: 10.1038/s41559-0170336-y. URL: https://doi.org/10.1038/s41559-017-0336-y.

[7] Daniel S Goldman. "On Topics of Thermodynamics, Complexity, Evolution, and Abiogenesis". In: (Feb. 2019). DOI: 10.31219/osf .io/4uczr URL: https://doi.org/10.31219/osf .io/4uczr.

[8] Jason A. Hodgson and Todd R. Disotell. "Anthropological Genetics: Inferring the History of Our Species Through the Analysis of DNA". In: Evolution: Education and Outreach 3.3 (Aug. 2010), pp. 387-398. DOI: 10.1007/s12052-010-0262-9. URL: https:// doi . org/10 .1007/ s12052-010-0262-9.

[9] Christophe Malaterre. "Can Synthetic Biology Shed Light on the Origins of Life?" In: Biological Theory 4.4 (Dec. 2009), pp. 357-367. Dor: 10 . 1162/biot_a_00002. URL: https://doi.org/10.1162/biot_a_00002.

[10] Addy Pross. "Toward a general theory of evolution: Extending Darwinian theory to inanimate matter". In: Journal of Systems Chemistry 2.1 (June 2011), p. 1. ISSN: 1759-2208. DOI: 10.1186/1759-2208-2-1. URL: https: //doi.org/10.1186/1759-2208-2-1.

[11] Anders Sandberg, Eric Drexler, and Toby Ord. "Dissolving the Fermi Paradox". In: (2018). eprint: arXiv: 1806.02404. 
[12] Sumantra Sarkar and Jeremy L. England. "Design of conditions for selfreplication". In: Physical Review E 100.2 (Aug. 2019). DOI: 10.1103/ physreve.100.022414. URL: https://doi.org/10.1103/physreve. 100.022414

[13] Howard A. Smith. "Alone in the Universe: Despite the growing catalog of extrasolar planets, data so far do not alter estimates that we are effectively on our own". In: American Scientist 99.4 (2011), pp. 320-327. ISSN: 00030996. URL: http://www.jstor.org/stable/23019382.

[14] Stephen Jay Gould, "Nonoverlapping Magisteria," 1997. URL: http:// www . stephenjaygould . org/library/gould_noma . html (visited on $12 / 24 / 2018)$.

[15] J. T. Trevors and M. H. Saier. "Three Laws of Biology". In: Water, Air, and Soil Pollution 205.1 (Jan. 2010), pp. 87-89. ISSN: 1573-2932. DOI: 10.1007/s11270-008-9925-3. URL: https : / / doi . org/10 . 1007/ s11270-008-9925-3

[16] Tom Westby and Christopher J. Conselice. "The Astrobiological Copernican Weak and Strong Limits for Intelligent Life". In: The Astrophysical Journal 896.1 (June 2020), p. 58. DOI: 10.3847/1538-4357/ab8225. URL: https://doi.org/10.3847\%2F1538-4357\%2Fab8225. 